\title{
A note on the acoustic environment in a usually quiet residential area after the 'state of emergency' declaration due to COVID-19 pandemic in Japan was lifted: supplementary survey results in post-emergency situations
}

https://doi.org/10.1515/noise-2020-0016

Received Aug 21, 2020; accepted Sep 03, 2020

\begin{abstract}
In a preceding report (UCL Open: Environment, 2020;1;6), an example of results on changes in the acoustic environment from a local-scale survey in a quiet residential area during and after the 'state of emergency' due to COVID-19 pandemic in Japan is presented: the noise level was 1-2 dBA lower during the state of emergency, which is smaller than reported from large cities. This note presents the results of a follow-up survey in the same area to provide some more examples to gain an insight into the acoustic environment in this area. In this note, the measurement results of noise levels in June 2020, a few weeks after the cancellation of the state of emergency, are mainly reported. As the results are almost the same as those from during the state of emergency, we can infer that either the noise level was reduced in June to a level that was almost the same as that during the state of emergency, or the noise level after its cancellation in May was possibly higher than usual. In either case, the change in noise level was small, and it was difficult to conclude which case was true.
\end{abstract}

Keywords: acoustic environment; COVID-19 pandemic; state of emergency; noise level; local-scale survey; quiet residential area

\section{Introduction}

The COVID-19 outbreak has brought a lot of changes and challenges to our daily life, and its impact on the envi-

\footnotetext{
${ }^{\star}$ Corresponding Author: Kimihiro Sakagami: Environmental Acoustic Laboratory, Department of Architecture, Graduate School of Engineering, Kobe University, Rokko, Nada, Kobe 657-8501, Japan; Email: saka@kobe-u.ac.jp
}

๑ Open Access. (C) 2020 K. Sakagami, published by De Gruyter. (CC) BY License ronment is now an emerging topic of paramount importance [1]. This includes its impact on the acoustic environment; for example, lockdowns due to the COVID-19 pandemic have been reported in a number of cases as resulting in a significant reduction of noise in large cities and mega-infrastructures such as airports [2-5]. A method of evaluation for noise levels and a taxonomy of reduction in such cases has also been proposed [6]. There have also been some local-scale studies on the impact of lockdown schemes, the results of which are somewhat different from those focused on busy areas and mega-infrastructures [7, 8]. In many countries, lockdown schemes have been lifted, and the people's daily lives seem to be getting back to normal. However, there are still many differences, e.g., social distancing and so-called 'new style' behaviours. Discussion on the impact of lockdowns on the urban acoustic environment is one thing, but now it is necessary to discuss what happened after the lockdown schemes were lifted. This note is intended to be a follow-up report to the author's previous work of a local-scale survey on the effect of the 'state of emergency' declaration from 7 April to 25 May 2020 [8]. A similar small-scale survey was made in June (almost a few weeks after cancellation of the state of emergency), and the results are compared herein with those found during the state of emergency and immediately after its cancellation in May 2020, to consider how the acoustic environment changed during this period.

In contrast to the drastic reduction of noise levels in busy and usually noisy areas reported from many countries, usually quiet residential areas have been discussed in only a limited number of studies $[7,8]$. In Japan in particular, lockdown in a strict sense was not made, but another scheme was employed. By this scheme, Government and local government requests for voluntary restraint on various activities were made in the form of the declaration of a 'state of emergency'. This was somewhat different from the 
lockdowns in other countries around the world, as it was a request for self-restraint that had no legal effect and was considered to be different in its impact on the acoustic environment as well. The author performed a limited smallscale study of the noise levels and types of sound sources during and after the state of emergency in a usually quiet residential area, and reported the results [8]. The results showed that, at least in the usually quiet area surveyed in the study, the reduction in noise due to the declaration of the state of emergency was about 1-2 dBA in $\mathrm{L}_{A e q}$ (Equivalent Continuous Noise Level), which is significantly different from the reported differences in noise observed in large cities and airports. In this example, the considerations were limited to a very specific area, and the results, which were short-term averaged noise levels, are limited. However, it can be considered that similar results may be obtained for other quiet residential areas.

Aletta et al. [7] conducted a more elaborated and complete study on the impact of lockdown on the local-level urban acoustic environment using short-term measures. They aimed to understand how lockdown measures have manifested at a local level to better determine how the person-level experience of the urban soundscape was affected and how those effects were different across urban space types. They chose 11 points in London, and shortterm (30-second) binaural recordings were obtained for the study; they compared the results between Spring 2019 (pre-lockdown) and Spring 2020 (during lockdown). According to their analyses, the average reduction in $\mathrm{L}_{\text {Aeq }}$ was $5.4 \mathrm{dBA}$, and the range was from 10.7 to $1.2 \mathrm{dBA}$. They pointed out that, as the multi-faceted consequences of the COVID-19 crisis unravel, it is also important to acknowledge that changes are happening at different scales. Also, they remarked that the improvement in terms of environmental noise pollution has been a positive unintended effect of the lockdown measures in many cities; planning for post-COVID scenarios is needed to make sure that sound levels do not go straight back to normal (or even worse) as soon as containment measures are relaxed.

The above studies revealed the impact of lockdown (and similar schemes) on urban acoustic environments at the local scale, which can be somewhat smaller than the reported noise level reduction from city-scale studies $[2,3,6]$.

In Japan, the state of emergency was lifted on 25 May 2020. However, almost all schools remained closed throughout May even after the state of emergency was lifted, and while social life showed a gradual tendency to return to normal, it is likely that the life patterns of children and students did not change significantly from those during the state of emergency.
From June, non-university schools were reopened; however, the situation changed from time to time because of half-day shifts and staggered classes, and it was difficult to ascertain the acoustic environment in this transitional state. This paper describes the results of a survey conducted in June 2020 (from 11 to 28 June, during the rainy season in Japan) when the sound environment had stabilised and school operations had normalised. The survey was based on the results of daily noise level variation determined from fixed-point observations, which showed that daily activities such as school reopening and commuting had almost normalised and became more or less the same as they were before the state of emergency.

A similar survey was also made from 19 July to $2 \mathrm{Au}-$ gust, which is shown in the Appendix, as the acoustic environment in this area was drastically changed by cicada sounds. Also, some results from a soundwalk survey in this area are shown in Appendix A as additional information.

The following are the results of measurements of the sound environment in this region with the sound pressure level of the region as a whole. Unlike the case of a large city centre, the results shown here are only an example of this region and are not intended to be generalised, since differences in behavioural patterns are likely to occur in residential areas depending on the attributes of the people living there and the surrounding environment. Rather, it is easy to imagine that each region has its own unique behaviour and that it is not easy to generalise the results from a specific area to others, but it is considered necessary to accumulate examples from various areas to gain insight.

\section{Methodology and results}

In the previous report [8], various measurements and surveys were performed; however, in the present study, fixedpoint measurement was the focus. The measurements were conducted at the same points as those previously reported [8], as shown in Figure 1. For the fixed-point observations, the equivalent noise level, $\mathrm{L}_{A e q}(\mathrm{dBA})$, was measured using a standardised (Class 2) sound level meter. The integration time was set to 1 minute in this study, although this was much shorter than that proposed by Asensio [6]. However, according to Aletta et al. [7], short-term measures are useful to soundscape studies in similar situations. Considering the results in [6], this time of $1 \mathrm{~min}$ was chosen (instead of $30 \mathrm{~s}$ ) because the measurements were made almost hourly and because the noise was almost stationary; therefore, it was difficult to show a time-specific difference in the average value for a long time in order to show a clear 


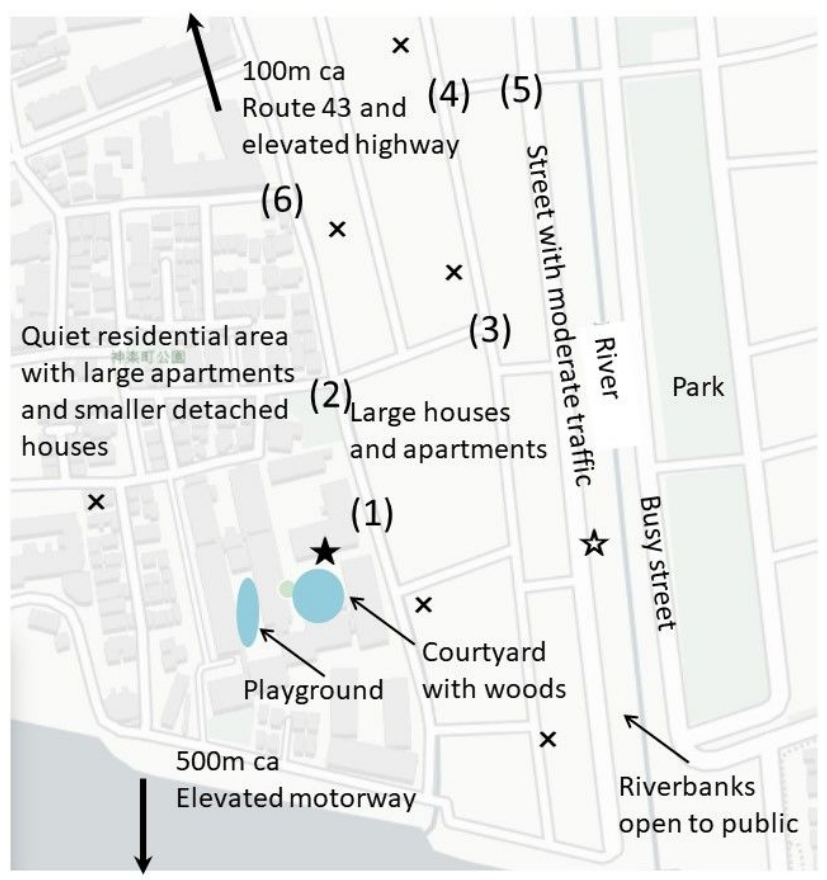

Figure 1: Map of the surveyed area (eastern edge of Kobe). The numbers (1) to (6) show measuring points in the previous survey [8]. The black star is the measuring point for fixed-point observations in both the present and previous studies. The white star is the measuring point for monitoring noise levels in a public space (riverbank). The $\mathrm{X}$ marks show construction sites (most of which were finished during the June survey reported herein).

time-specific change. The purpose of this survey is to understand the subsequent changes in the area covered by the previous report, and it is desirable in this case to use a method similar to that used in the previous report.

Regarding observation points 1 to 6 , in this study, a supplemental soundwalk survey was performed to provide an overview of the acoustic environment in this area. For this purpose, NoiseCapture on an Android tablet (Teclast P80X) was used. The technical information of the NoiseCapture app is available on its web site [9] and documented in [10]. Before the measurements, the app and the device were calibrated using a standardised sound level meter (SLM) (Class 1), and its validity, confirmed via the method in [11, 12], was reported in [8]. The results of a sound map made via soundwalk with this app give the noise level range $\left(\mathrm{dBA}, \mathrm{L}_{\text {Aeq }}\right)$ along the soundwalk course. Therefore, exact values of each point are not given, but one can find the range as well as noisy or quiet areas from the map. The results of the survey are presented later in this note as additional information on the surveyed area.

The point marked with a white star on the map was not used in the present study, because the riverbank was no longer so crowded as it was during the surveyed period in the previous study [8].

Sound source observations were not performed in the main survey in the fixed-point measurements. This is because there was no significant difference between the previous survey in May (during and after the state of emergency) and that in June (nearly a few weeks after the cancellation of the state of emergency declaration). Also, in July-August, the acoustic environment in the area was dominated by cicada sounds, which masked most other sounds.

Other specific details of each survey are described in the corresponding sections.

\subsection{Day history of the noise level in June}

The $\mathrm{L}_{\text {Aeq }}$ value was measured basically every hour every day from 11 to 28 June 2020. The results were classified into Dawn (04:00-06:00), Morning (07:00-11:00), Afternoon (12:00-16:00), Evening (17:00-19:00), and Midnight (23:00-01:00) according to people's life patterns. Then, the results obtained in a timeslot were averaged in each time category, e.g., results obtained during 07:00-11:00 were averaged and allocated to Morning. The results obtained in this way are summarised for weekdays and weekends (Saturday and Sunday) separately in Figure 2.

On both weekdays and weekends, the noise level was low at dawn and rose gradually, then fell to a low level in the evening, and was considerably quieter late at night. There was little difference between weekdays and weekends, and no statistical significance was found: $t(87)=0.67$, $p=0.5$. However, there was a relatively large difference only in the morning. This is likely due to the fact that many

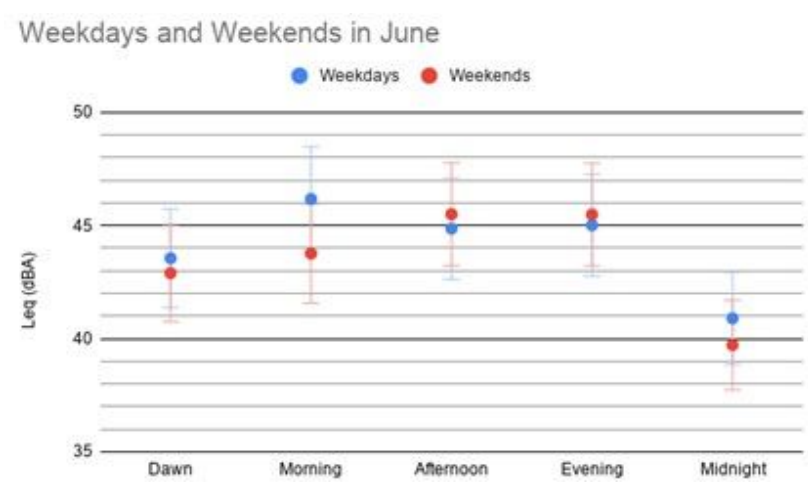

Figure 2: Comparison of the day history of noise levels $\left(\mathrm{L}_{\text {Aeq }}, \mathrm{T}=1\right.$ $\mathrm{min}$ ) on weekdays (blue) and weekends (red) in June. The plot for each time block is the averaged value over all weekdays and weekends during the observation period. Error bars are also indicated (95\% range). 
families have a high level of activity on weekdays as they prepare for work and school in the mornings, whereas on weekends there is relatively less activity and a lower noise level because there is no need to do so.

In terms of perceived sounds, since this area is usually very quiet, it is difficult to point out a dominating or outstanding sound source, however, in the early morning, the only sounds were almost exclusively bird calls. The faint road traffic noise from the rather distant national road was heard almost always, however no other traffic noise was heard except for occasional passing by of vehicles in the street nearby. No other activity sounds were heard in the early morning. In the morning, household noise was heard early on, but this decreased as the morning progressed. In the afternoon, there was generally less noise, but in the evening, the sounds of children and students returning from school and their activities were heard. In the late evening, other than road traffic noise from the national highway, most of the noise was from the mechanical ventilation of homes and boilers. Occasional construction noise was also heard, but most of the work seems to have been completed in the morning, and such noise was less common in the afternoon. Also, in the last week of June, occasional air-conditioner noise was heard, but it was not significantly strong. Therefore, it can be summarised that, bird calls in the early morning and voice of children in the early evening were mainly perceived as the sounds characterising the acoustic environment in this area. As June is rainy season, most days during the survey were either cloudy or rainy. The measurement point is surrounded by many trees, and rain noise had some effect due to rainfall hitting the vegetations, which is somewhat unusual in the other seasons. Therefore, the measurement was not carried out when it was heavily and mediumly raining. The ground surface around the measurement point was mostly paved by asphalt, except for the courtyard and vegetation areas.

Figure 3 shows a comparison of the noise levels during the state of emergency, immediately after its cancellation, and in June (nearly a few weeks after the cancellation). Note that the data from the previous report represented every one-hour period, but here they were reorganised into the categories used in the present work and averaged in each category. In the previous report, it was shown via t-test that the noise levels during the state of emergency and after its cancellation significantly differed. However, here, by this reorganisation of the data, the significance of this difference was lowered: $t(8)=1.57, p=0.16$ (using the reorganised and averaged data) which is interpreted as weak significance). This reorganisation of the data allows for comparisons of the rough trends of each data set. (For
During and after state of emergency and in June

During state of emergency (13-24 May 2020) After its cancellation (25-28 May 2020) A few week after state of emergency (11-28 June 2020)

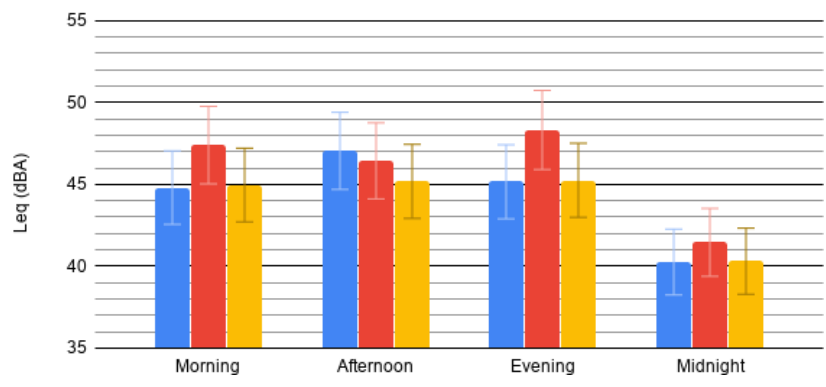

Figure 3: A comparison of the noise levels during the state of emergency, immediately after its cancellation, and in June. Error bars indicate the $95 \%$ ranges of each value.

reference, by using unprocessed data t-test shows: $t$ (209) $=2.58, p=0.01$ ).

The day history of the noise level in June is inferred to be close to that in the state of emergency (a t-test by using the reorganised average data: $t(8)=0.30, p=0.77$; though $t(203)=1.9$, and $p=0.06$ with unprocessed data before reorganisation). The only observable difference between them is in the afternoon, where the noise level in June was exclusively lower than the others. In June, it is supposed that the town became quieter because children and students were in school.

On the other hand, the day history immediately after the cancellation of the state of emergency is exclusively larger than the others. The t-test with the results in June shows strong significance: $t(8)=2.78, p=0.02$ with reorganised and averaged data, and $t(209)=2.55, p=0.00005$ with unprocessed data before reorganisation; it shows especially clear differences in observed noise levels in the morning, afternoon, and evening. This means that the noise level was reduced in June to a level that was almost the same as that during the state of emergency. In other words, the noise level immediately after the cancellation of the state of emergency in May was possibly higher than usual. In any case, it is highly probable that the difference between the level after the cancellation of the state of emergency and that in June resulted from the difference in the operation of schools; for example, in the early evenings, the noise level became lower in June, which is inferred to be the result of fewer children playing outside during these hours. Also, June is the rainy season in Japan, and rain could prevent people from going outside, making the area quieter. 


\subsection{Additional information from the soundwalk survey}

For measurement points 1 to 6 from the previous report, NoiseCapture (installed on an Android tablet, Teclast $\mathrm{P} 80 \mathrm{X}$ ), which was also used in the previous report [8], was employed. It was calibrated and checked for accuracy using a Class 1 sound level meter (SLM). For details of the accuracy check results, see [8], and for the method used for the accuracy check, see [11, 12]. The overall noise level and characteristics of the surveyed area and how these changed after the declaration of the state of emergency was lifted were investigated by way of a soundwalk using NoiseCapture.

In the previous report [8], it was reported that the noise level usually ranged from 45 to $65 \mathrm{dBA}$, the higher levels of which were observed only when cars passed by, in the pre-pandemic period (winter 2019). It seemed to increase slightly during and after the state of emergency due to increased traffic volume, although the difference was not statistically significant. Since the COVID-19 outbreak began, people have tended to either stay home working remotely or commute by private vehicle to avoid public transport. In the post-emergency situation, the traffic volume still seems to have not reduced to its level prior to the state of emergency.
Figure 4 shows the results of the soundwalk survey conducted nearly two months after the state of emergency was lifted (20 July 2020). The noise level range was almost the same as that in the previous surveys [8]. In (a), some red marks indicate high noise levels due to cicada sounds, which were especially high in the morning. However, in (b) and (c), most of the red marks correspond to cars and motorbikes passing by. Considering these results, the acoustic environment was not much different from that in winter 2019.

\section{Concluding remarks}

This note presents the results from a small-scale survey of the acoustic environments in June and July 2020 in a residential area that was surveyed during the state of emergency in April-May 2020 due to the COVID outbreak [8]. In the previous report, the reduction in noise levels due to the state of emergency declaration was discussed, and this reduction was reported to be approximately $1-2 \mathrm{dBA}$ in the area. A similarly small reduction was reported by Aletta et al. [7]. In their work, they compared the noise levels during lockdown with those in 2019 (pre-lockdown). However, although in [8], data taken from 2019 were also referenced,
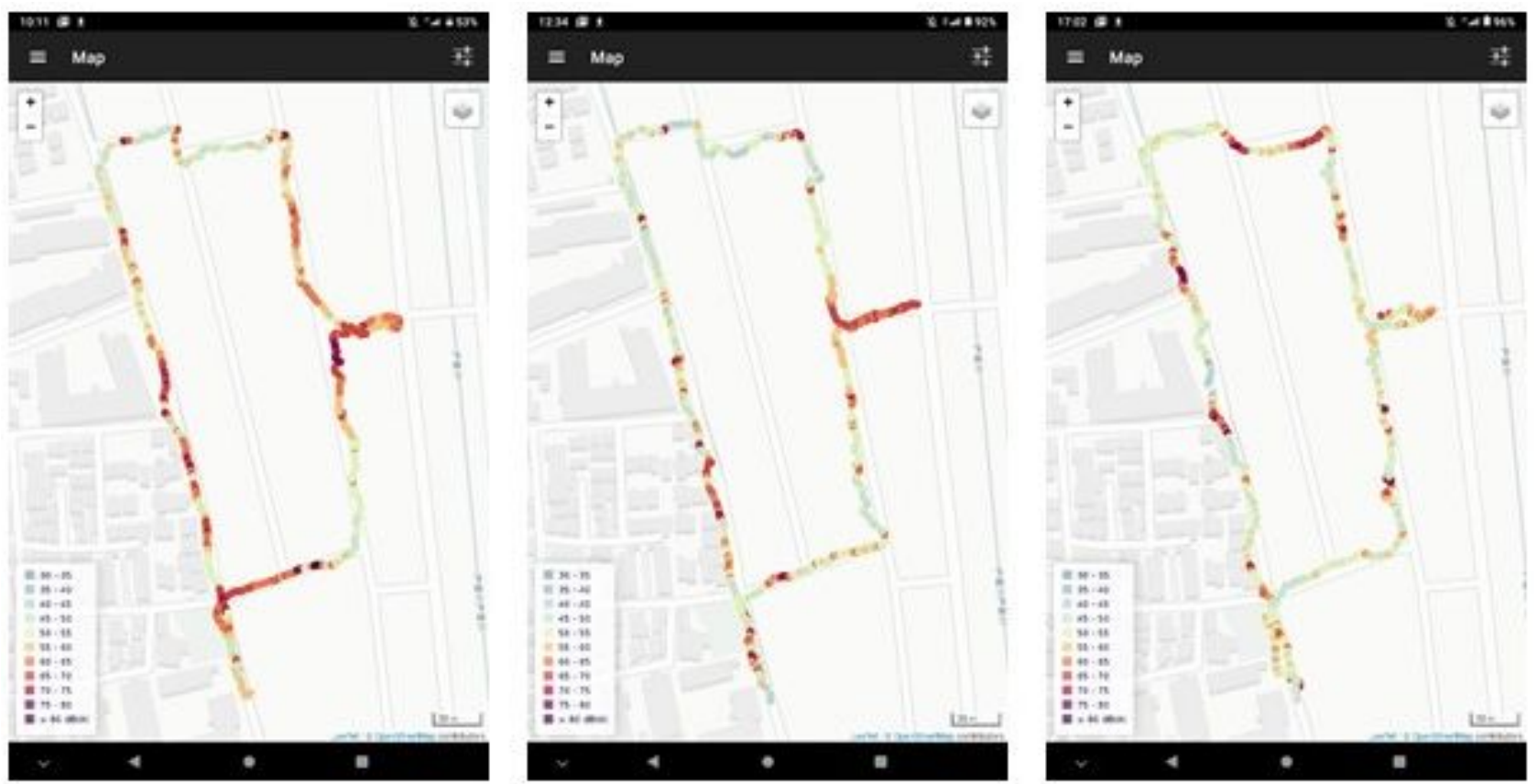

Figure 4: Noise maps taken from the results of soundwalks on 20 July 2020 using Noise Capture. This route includes Points (1) to (6) and the surrounding area. (a) 09:40-45; (b) 12:10-15; (c) 16:40-45. Red marks indicate noise levels higher than $60 \mathrm{dBA}\left(\mathrm{LA}_{e q}\right)$. The areas with higher levels were affected by cicada sounds in the morning (a). However, in many cases, these areas were somewhat quiet at noon and in the evening ( $b$ and $c$ ) when the cicadas were not active. In maps (b) and (c), the red zones are mainly affected by cars passing by. 
the main finding was that the noise level was smaller during the state of emergency than after it was lifted.

The results from the measurements made in June (a few weeks after the cancellation of the declaration) were close to those from during the state of emergency. This can be considered in two ways: one is that the noise levels returned to the lower level during the state of emergency, and the other is that the noise level immediately after the cancellation of the state of emergency was higher than usual, because of rather unusual behaviour of residents due to school closures. The noise levels in each area fluctuate according to various factors, such as human behaviours, animals, vegetation, etc. Therefore, it is difficult to define what the 'usual' acoustic environment is; however, at least in the specific area surveyed in this smallscale study, the change in the noise level was small in any case, and there may be only a small possibility for the level to change due to the impact of a pandemic, etc.

As, however, this is only an example and cannot be generalised, it may be necessary to consider what the usual acoustic environment is or how it should be, if one should take this opportunity presented by the COVID outbreak as a motivation to reconstruct the acoustic environment in urban areas.

Funding: This work received no external funding.

Conflict of Interests: There is no conflict of interest regarding the publication of this paper.

Author contribution: All work was carried out by the author (KS).

\section{References}

[1] Aletta F., Osbon D., The COVID-19 global challenge and its implications for the environment - what we are learning, UCL Open Environ., 2020, 1, 5. DOI: 10.14324/111.444/ucloe.000008
[2] Arenas J.P., Acoustics and vibration in the time of the pandemic, Int. J. Acoust. Vib., 2020, 25(2), 131-132. DOI: 10.20855/ ijav.2020.25.2E96

[3] Bruit et confinement: Acoucité objective les évolutions du paysage sonore urbain en France. Le CIDB [online]. 4 May 2020. Available from: https://www.bruit.fr/bruit-et-politique/ confinement (in French) (Accessed 17 Aug 2020)

[4] Acoucité, Impact sur l'environnement sonore (Ver. 1, $29 \mathrm{Apr}$ 2020). Available from: https://www.bruit.fr/images/2020/04/ 28/Impact_COVID-19_sur_IEnvironnement_Sonore_V1.pdf (in French). (Accessed 17 Aug 2020)

[5] Aletta F., Brinchi S., Carrese S., Gemma A., Guattari C., Mannini L. et al., Analysing urban traflc volumes and mapping noise emissions in Rome (Italy) in the context of containment measures for the COVID-19 disease, Noise Mapp. 2020, 7(1), 114-122. DOI: 10.1515/noise-2020-0010

[6] Asensio C., Aumond P., Can A., Casco L., Lercher P., Wunderi J-M. et al., A taxonomy proposal for the assessment of the changes in soundscape resulting from the COVID-19 lockdown. Int. J. Environ. Res. Pub. Health, 2020, 17, 4205. DOI: 10.3390/ijerph17124205

[7] Aletta F., Oberman T., Mitchell A., Tong H., Kang J., Assessing the changing urban sound environment during the COVID-19 lockdown period using short-term measurements, Noise Mapp. 2020, 7, 123-134. DOI:10.1515/noise-2020-0011

[8] Sakagami K., How did the 'state of emergency' declaration in Japan due to COVID-19 pandemic affect the acoustic environment in a rather quiet residential area? UCL Open Environ., 2020, (1)06. DOI: 10.14324/111.444/ucloe.000009

[9] NoiseCapture page in Noise Planet. Available from: https:// noiseplanet.org/noisecapture (Accessed 17 Aug 2020)

[10] Picaut J., Fortin N., Bocher E., Petit G., Aumond P., Guillaume G., An open-science crowdsourcing approach for producing community noise maps using smartphone, Build Environ., 2019, 148, 20-33. DOI: 10.1016/j.buildenv.2018.10.049

[11] Sakagami K., Satoh F., Omoto A., Revisiting acoustics education using mobile devices to learn urban acoustic environments: Recent issues on current devices and applications, Urban Sci., 2019, 3, 73. DOI: 10.3990/urbansci3030073

[12] Sakagami K., Satoh F., Omoto A., Use of mobile devices with multifunctional sound level measurement applications: Some experiences for urban acoustics education in primary and secondary schools, Urban Sci., 2019, 3, 111. DOI: 10.3990/urbansci3040111 


\section{Appendix A: Supplementary results and observations from measurements made in July-August}

In July, due to the large number of trees in the area, the noise level from cicadas was very high. As a result, the noise level was exclusively determined by the sound of cicadas (Figure A1).

The sound of cicadas determined the noise level almost exclusively on both weekdays and weekends, with extremely high noise levels in the morning when cicadas are more active. When the temperature rose above a certain level, the noise level quietened down, but it was still

Weekdays and Weekends in July-August

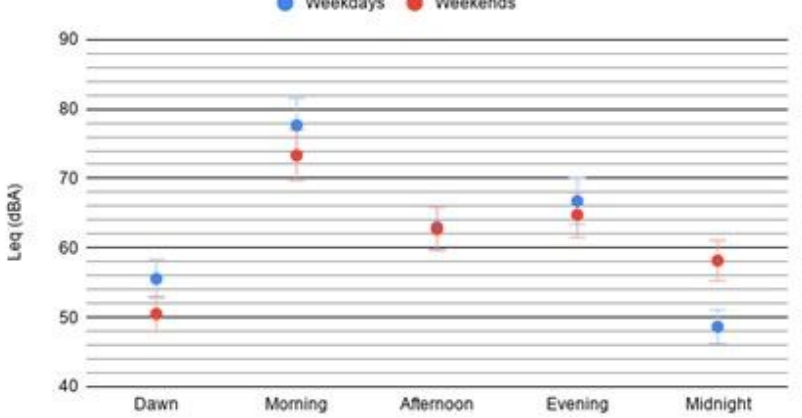

Figure A1: Comparison of the day history of the noise levels $\left(\mathrm{L}_{\text {Aeq }}, \mathrm{T}\right.$ $=1 \mathrm{~min}$ ) on weekdays (blue) and weekends (red) in July-August (19 July to 2 August 2020). The plot of each time block is the averaged value over all weekdays and weekends during the observation period. Error bars are also indicated ( $95 \%$ range). Note that the significant difference was not observed by t-test between Weekdays and Weekends: $t(57)=0.18, p=0.86$.

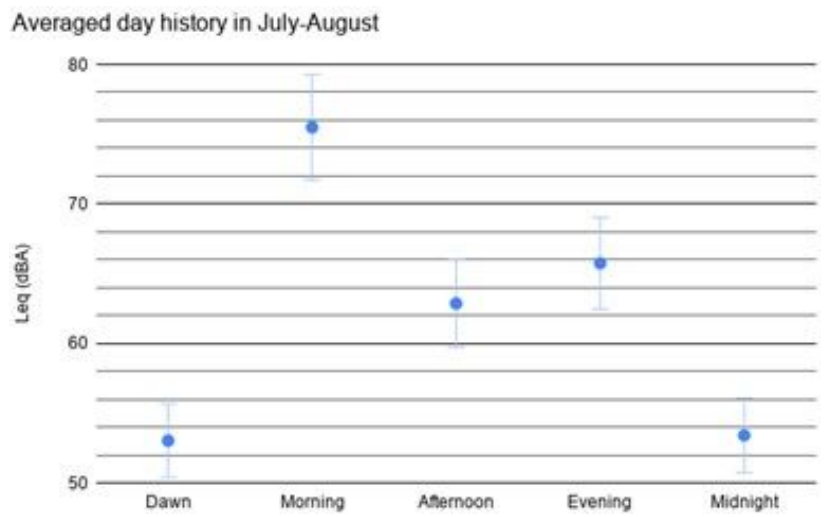

Figure A2: A comparison of the day history of the averaged noise levels $\left(\mathrm{L}_{\text {Aeq }}, \mathrm{T}=1 \mathrm{~min}\right)$ over both weekdays and weekends in JulyAugust. The plot of each time block is the averaged value over all weekdays and weekends during the observation period. Error bars are also indicated ( $95 \%$ range). quite high throughout the day. The cicadas' activity is influenced by the temperature and weather conditions, and cicada activity seems to have been particularly high during the measurement period due to high daytime temperatures; however, there is little qualitative difference in the change trend, and the values are similar, i.e., low at Dawn and in the Evening, and high in the Morning and Afternoon. Exceptionally, Midnight noise levels were particularly high on weekends because there were days when it was particularly hot and cicadas were active until late at night. Therefore, Figure A2 shows the averages for weekdays and weekends combined. This makes the above trend even clearer.

Comparing the results for June and July together (Figure A3), we can see that although the noise level was overwhelmingly higher in July, the change trends throughout the day were generally similar, with low levels at dawn and at night and higher levels during the daytime. The large peak in the morning in July is because this was the most active time for cicadas. However, excluding these fluctuations due to cicadas, the rough trends of the noise level day history can be assumed to be the same in residential areas like this and in any season if only qualitative trends are considered.

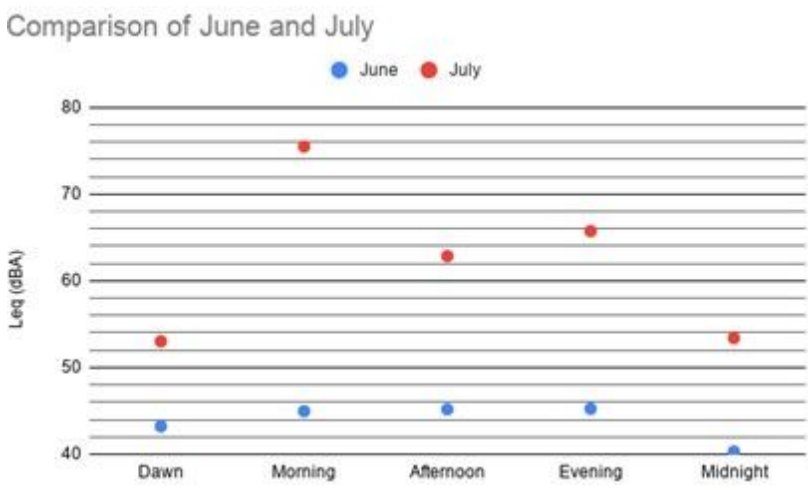

Figure A3: Comparison of the day history of the averaged noise levels from June measurements (blue) and July-August measurements (red).

These examples cannot be used in a discussion to evaluate the effect of the COVID outbreak on the acoustic environment in this area as they are strongly dominated by cicada sounds. However, this should be considered a 'usual' environment every summer in this area with many trees. 